\title{
Upgrade of the MAGNEX spectrometer toward the high- intensity phase of NUMEN
}

\author{
Manuela Cavallaro ${ }^{11}$, Clementina Agodi ${ }^{1}$, Giuseppe A. Brischetto ${ }^{1,2}$, Salvatore Calabrese ${ }^{1}$, Daniela Calvo $^{3}$, Vittoria \\ Capirossi $^{3,4}$, Francesco Cappuzzello ${ }^{1,2}$, Diana Carbone ${ }^{1}$, Irene Ciraldo ${ }^{1,2}$, Franck Delaunay ${ }^{1,2,5}$, Paolo Finocchiaro ${ }^{1}$, \\ Maria Fisichella ${ }^{1}$, Felice Iazzi ${ }^{3,4}$, Federico Pinna ${ }^{3,4}$, Diego Sartirana ${ }^{3}$, Alessandro Spatafora ${ }^{1,2}$, Onoufrios Sgouros ${ }^{1}$, \\ Vasilis Soukeras ${ }^{1,2}$, Domenico Torresi ${ }^{1}$, Salvatore Tudisco ${ }^{1}$
}

For the NUMEN collaboration

${ }^{1}$ INFN - Laboratori Nazionali del Sud, Catania, Italy

${ }^{2}$ Dipartimento di Fisica e Astronomia "Ettore Majorana", University of Catania, Catania, Italy

${ }^{3}$ DISAT, Politecnico di Torino, Torino, Italy

${ }^{4}$ INFN - Sezione di Torino, Torino, Italy

${ }^{5}$ LPC Caen, Normandie Univ, ENSICAEN, UNICAEN, CNRS/IN2P3, Caen, France

\begin{abstract}
The NUMEN experimental activity with accelerated beams is performed at INFN-Laboratori Nazionali del Sud (LNS) in Catania using the Superconducting Cyclotron and the MAGNEX magnetic spectrometer. The scientific motivation of NUMEN is to extract experiment-driven information on the nuclear matrix elements entering in the expression of the $0 v \beta \beta$ decay half-life. The reaction cross sections involved, especially for the double charge exchange process, are very low, thus limiting the present exploration to a few selected isotopes of interest in the context of typically low-yield experimental runs. In order to make feasible a systematic study of all the candidate nuclei, a major upgrade of the LNS facility is foreseen to increase the experimental yield by more than two orders of magnitude. To this purpose, frontier technologies are being developed for the accelerator and the detection systems. An updated description of the choices derived from the recent R\&D activity on the target system and MAGNEX focal plane detector is given.
\end{abstract}

\section{Introduction}

MAGNEX [1] is a large acceptance magnetic spectrometer installed at INFN-Laboratori Nazionali del Sud in Catania (Italy). It analyses in momentum and detects the products of different kinds of nuclear reactions induced by ion beams from both the Tandem Van der Graaff and Superconductive Cyclotron (CS) accelerators. Since 2014 it has been the main facility used for the NUMEN (NUclear Matrix Elements for Neutrinoless double beta decay) project [2].

NUMEN aims at providing experiment-driven information on the Nuclear Matrix Elements (NME) involved in the expression of the decay half-life of neutrinoless double beta decay $(0 \nu \beta \beta)$, by measuring the cross-sections of nuclear Double Charge Exchange (DCE) reactions [3], [4]. However, the DCE cross sections are very small (a few $\mathrm{nb}$ ), thus requiring a high sensitivity [5]. For this reason, as discussed in [2], the present limits of the facility in terms of beam power delivered by the CS accelerator and the acceptable rate of a few $\mathrm{kHz}$ for the MAGNEX focal plane detector (FPD) have limited so far the exploration of DCE to a small number of cases (e.g., ${ }^{12} \mathrm{C},{ }^{40} \mathrm{Ca},{ }^{48} \mathrm{Ti},{ }^{76} \mathrm{Ge},{ }^{76} \mathrm{Se}$, ${ }^{116} \mathrm{Cd},{ }^{116} \mathrm{Sn},{ }^{130} \mathrm{Te}$ ) with beam power of a few $\mathrm{W}$. The systematic exploration of all the nuclei of interest to $0 \nu \beta \beta$ decay, as foreseen in NUMEN, requires an upgraded set-up able to work with $\mathrm{kW}$ beam power [6]. Thus, NUMEN has triggered the current upgrade of the INFN-LNS CS and associated infrastructures to develop such high-intensity ion beams.

An intense R\&D activity in beam transport, target technology, mechanical integration, detectors and electronics has developed in the last years and is ongoing within the NUMEN project.

In the present paper, an overview of the different activities undertaken for the upgrade of the MAGNEX spectrometer in preparation of the high-intensity phase of NUMEN is given. A picture of the MAGNEX spectrometer in its present, pre-upgrade state is shown in Fig. 1.

${ }^{1}$ Corresponding author: manuela.cavallaro@Ins.infn.it 


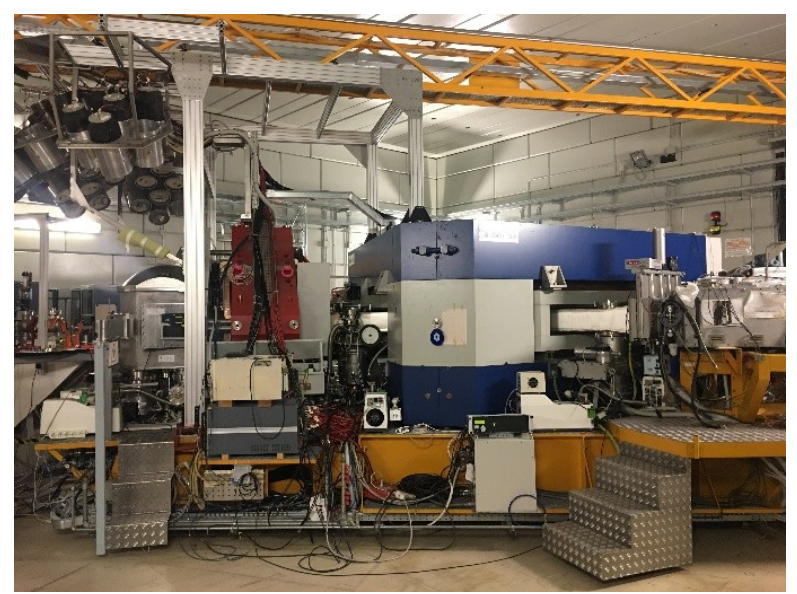

Fig. 1. View of the MAGNEX spectrometer at INFN-LNS

\section{The upgrades}

\subsection{Development of targets for high-intensity beams}

The goal of the target development activity is to design and produce a number of thin solid targets of specific isotopes, that will be irradiated with intense ion beams during the NUMEN experiments.

The main issues are related to the thickness of the target, which affects the energy resolution of the reaction products, and the dissipation of the heat produced in the target by the beam energy loss. The proposed solution is to prepare each target by depositing a film of the isotope of interest, with a thickness of a few hundred $\mu \mathrm{g} / \mathrm{cm}^{2}$, on a $2-\mu \mathrm{m}$ thick HOPG (Highly Oriented Pyrolithic Graphite) backing, which will withstand the target layer, fully strip the reaction products, and quickly transfer the heat to a target cooling system [7].

During the R\&D phase, the evaporation techniques have been explored and their parameters (temperature, duration, use of a buffer, buffer material, etc.) have been optimized for a few target materials, with the aim of minimizing the thickness non-uniformity and rugosity which can degrade the energy resolution [8]. Moreover, the target cooling technique has been studied and optimized, evaluating, for each target, the maximum allowed ion beam intensity to avoid melting [9].

Fig. 2 shows a sketch of the target holder [10] to be mounted in the MAGNEX scattering chamber during the experimental run of NUMEN phase 4. Its shape has been designed to facilitate the heat flow toward the cold finger located at the bottom. In particular, the base is wide to ensure a large contact surface with the cold finger of the cryocooler. The top part is thin to guarantee a wide solid angle aperture for the ancillary detectors surrounding the target (e.g. $\gamma$-ray calorimeter). The isotopically enriched target will be located at the bottom position. Three more target positions are present to allocate a pure HOPG target for background evaluation, an empty hole for the beam transport procedures and an alumina layer needed for the beam optics optimization.

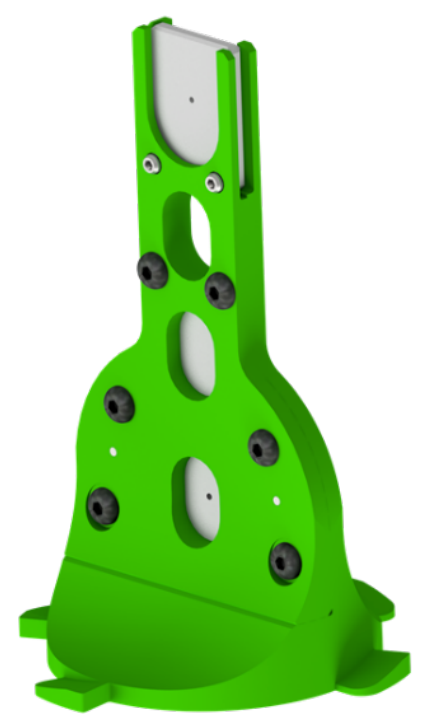

Fig. 2. Sketch of the NUMEN target holder

The target characterization methods have also been the subject of R\&D studies [8]. The determination of the thickness of the target and HOPG substrate and of their uniformity is of crucial importance. Several analysis techniques have been adopted. Rutherford Backscattering Spectroscopy (RBS) and Alpha Particle Transmission (APT) have been exploited to measure the thickness and the uniformity of the target layer and substrate. Both techniques can provide accurate evaluations of the average thickness of the sample, but only with APT can the thickness uniformity be directly quantified. The thickness obtained by APT can be verified with RBS, while the roughness of the isotopic deposition can be evaluated with Field Emission Scanning Electron Microscopy, which provides images of the sample surface topography.

A picture of the vacuum chamber used for APT measurements at INFN-LNS is shown in Fig. 3. A rotating plate divided in 4 sections, which allocate several target frames is visible, together an alphaparticle source and a silicon detector used to measure the residual energy of the alpha-particles going through the target. 


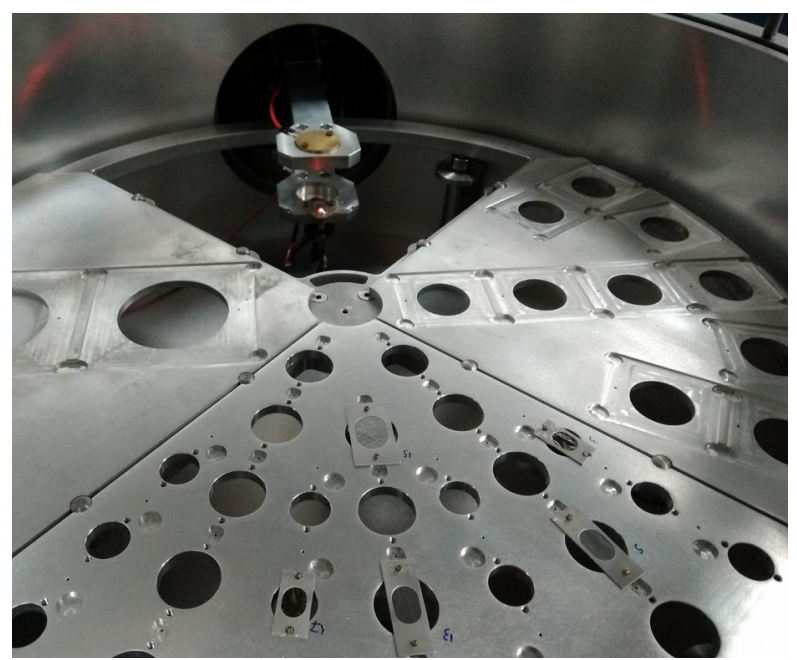

Fig. 3. Alpha-particle transmission setup at INFN-LNS

\subsection{The gas tracker system}

A gas tracker for the focal plane detector of the MAGNEX spectrometer is under development within the NUMEN project [11], [12]. The role of the tracker is to perform a three-dimensional measurement of the reaction ejectile trajectories crossing the focal plane. Such an operation is needed for particle identification purposes and to allow the high order reconstruction of the trajectories. Two main characteristics are required. The first is a high-resolution measurement of the phase space parameters of the ion tracks at the focal plane: $X_{\text {foc }}, Y_{\text {foc }}, \theta_{\text {foc }}, \varphi_{\text {foc }}$, where $X_{\text {foc }}$ is the horizontal coordinate (dispersive direction along the focal plane), $\theta_{\text {foc }}$ the horizontal angle, $Y_{\text {foc }}$ the vertical coordinate and $\varphi_{\text {foc }}$ the vertical angle. The required resolution is better than $0.6 \mathrm{~mm}$ for $X_{\text {foc }}$ and $Y_{\text {foc }}$ and lower than $5 \mathrm{mrad}$ for $\theta_{\text {foc }}$ and $\varphi_{\text {foc. }}$. Such a precise and accurate particle ray reconstruction is mandatory for the determination of the momentum vector (scattering angle and kinetic energy) of the ejectiles at the target position [13]. The second requirement is the ability to withstand the expected high rate of impinging particles (about 50 $\mathrm{kHz} / \mathrm{cm})$.

The tracker has an active volume of $1200 \times 150 \mathrm{x}$ $108 \mathrm{~mm}^{3}$, like the present one [14], [15]. A reduced size prototype, shown in Fig. 4, has been developed and built during the R\&D phase of NUMEN. This prototype, with mechanical and electrical features very similar to those of the final tracker, except for a shorter length which allowed easier tests in a small gas chamber. The applied voltages, the gas pressure and flowing system, the multiplication technology, and the front-end electronics are among the main features tested with the reduced-size prototype.
The prototype, as well as the full-size detector, works as a time projection chamber and consists of three main sections:

- a drift region, which is the active volume of the detector, crossed by the ejectiles of interest,

- an electron multiplication stage, based on multiple Thick Gas Electron Multipliers (mTHGEM),

- a segmented read-out electrode.

The incident charged particle crosses a thin mylar window (typical thickness between 1.5 and $6 \mu \mathrm{m}$ ) and leaves a track of ionized atoms and electrons in the low-pressure gas of the drift region between the cathode and the electron multiplication stage. Under the uniform electric field, the electrons drift with constant velocity toward the m-THGEM. As they reach the multiplication element, electrons are guided into the holes by the strong electric field. The produced electron avalanches reach the segmented read-out electrode, where the projection of the particle trajectory on the horizontal plane can be reconstructed. By the measurement of the drift time of the electrons in the gas, the projection of the trajectory on the vertical plane can also be reconstructed.

Detailed results from the prototype characterization in terms of measured currents and of different segmentation geometries for the anodic read-out plate are given in [11], [12].

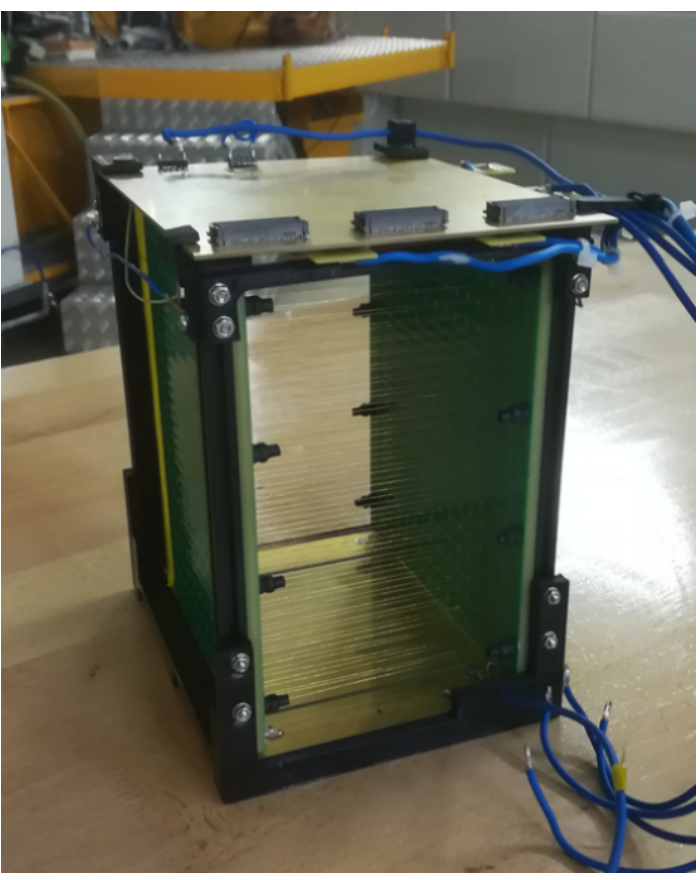

Fig. 4. View of the reduced-size tracker prototype. 


\subsection{The particle-identification wall}

The new gas tracker of the MAGNEX spectrometer is not designed to provide accurate information on ion energy loss. Consequently, particle identification (PID) must be demanded from a dedicated wall of telescope detectors downstream of the tracker. Fig. 5 shows the schematic layout of the PID wall system.

The most important requirements for the design of a detection system capable of unambiguously identifying ions in the region of interest for NUMEN ejectiles (typically $\mathrm{O}, \mathrm{F}$ and $\mathrm{Ne}$ ) are:

- radiation hardness (the heavy-ion fluence rate will be of the order of $10^{11} \mathrm{ions} / \mathrm{cm}^{2} \cdot \mathrm{yr}$ ),

- energy resolution (better than $2 \%$, to maintain the present performance in terms of atomic number and mass identification [16] and sensitivity in the cross-section measurements [5]),

- time resolution $(\sim 5 \mathrm{ns,} \mathrm{to} \mathrm{guarantee} \mathrm{an}$ accurate measurement of the drift time of the primary electrons in the gas tracker),

- degree of segmentation (to keep the pile-up probability below 3\% in the whole FPD),

- geometrical efficiency (which should be high enough to obtain an accurate measurement of the absolute cross section and to reduce the background from events with partial charge collection),

- detector thickness (which must stop the ejectiles of interest in a wide dynamical range of incident energies, typically 15 to 60 $\mathrm{MeV} / \mathrm{u}$ ),

- working in a low-pressure gas environment (typical pressure 10-100 mbar).

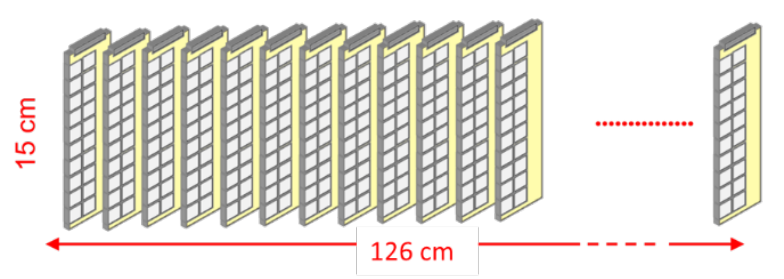

Fig. 5. Schematic layout of the PID-wall telescopes.

The R\&D activity on the PID wall resulted in the choice of an array of two-stage telescopes of Silicon Carbide ( $\mathrm{SiC}$ ) [17] and Thallium-doped Cesium Iodide $\mathrm{CsI}(\mathrm{Tl})$ detectors. The active area of each element is $1.5 \times 1.5 \mathrm{~cm}^{2}$, with $0.2 \mathrm{~mm}$ dead space between adjacent cells. The $\mathrm{SiC}$ detector is $100-\mu \mathrm{m}$ thick, while the $\mathrm{CsI}(\mathrm{Tl})$ scintillator is $5-\mathrm{mm}$ thick and is coupled to a Hamamatsu S3590 photodiode with a $1 \times 1 \mathrm{~cm}^{2}$ area. The capabilities of the system in terms of radiation hardness and mass resolution are discussed in [12]. A scheme of the mechanical arrangement of the telescopes is presented in Fig. 5, as a result of an optimization of the detection efficiency and of the mechanical integration [11].

\section{Conclusions}

The NUMEN project has promoted a major upgrade of the INFN-LNS research facility allowing for a significant increase of the beam intensity, which is needed for the systematic exploration of the DCE transitions of interest for $0 \nu \beta \beta$. Several aspects of the technology involved in heavy ion collision experiments demand for challenging $R \& D$.

In addition to the on-going improvement of the CS accelerator extraction technique for heavy ion beams in the regime of $\mathrm{kW}$ power and at energies from 15 to 70 $\mathrm{MeV} / \mathrm{u}$, crucial upgrades involve the MAGNEX spectrometer.

Detailed descriptions of such activities and of the proposed solutions are given in the NUMEN Technical Design Report [18]. Here an overview of the main results has been given, with a special focus on the target technology, on the focal plane detector tracker and particle identification wall.

This project has received funding from the European Research Council (ERC) under the European Union's Horizon 2020 research and innovation programme (grant agreement No 714625).

\section{References}

1. F. Cappuzzello et al., Eur. Phys. J. A 52, 167 (2016)

2. F. Cappuzzello et al., Eur. Phys. J. A 54, 72 (2018)

3. H. Lenske et al., Prog. Part. Nucl. Phys. 109 103716 (2019)

4. E. Santopinto, et al., Phys. Rev. C 98:061601 (2018)

5. S. Calabrese et al., Nucl. Inst.Method Phys. Res. A 980:164500 (2020)

6. C. Agodi et al., Universe 7, 72 (2021)

7. F. Iazzi, et al., WIT Trans. Engin. Sci. 116, 61 (2017)

8. V. Capirossi et al., Acta Phys. Pol. B 51, 661. (2020)

9. F. Pinna et al., Acta Phys. Pol. B 51, 665 (2020) 
10. D. Sartirana et al., Adv. Serv. Indust. Robot. 84, 533 (2020)

11. F. Cappuzzello et al., Front. In Astr. And Space Sc. 8, 668587 (2021)

12. P. Finocchiaro et al., Universe 6, 129 (2020)

13. F. Cappuzzello et al., Nucl. Instr. And Meth. A 638, 74 (2011)

14. M. Cavallaro et al., Eur. Phys. J. A 48, 59 (2012)

15. D. Torresi et al., Nucl. Instr. Method A 989, 164918 (2021)

16. F. Cappuzzello et al., Nucl. Instr. And Meth. A 621, 419 (2010)

17. S. Tudisco et al., Sensors 18, 2289 (2018)

18. F. Cappuzzello et al., Journ. of Modern Phys. A, submitted. 